\title{
Elementos del conocimiento especializado del profesor de matemáticas que se requieren para atender situaciones afectivas en el aula
}

\section{Elements of the mathematics teacher's specialized knowledge that are used to attend affective situations in the classroom}

\author{
Verónica Aguilar Mendieta, \\ Benemérita Universidad Autónoma de Puebla, México (veronica.aguilarm@alumno.buap.mx) \\ Eric Flores-Medrano, \\ Benemérita Universidad Autónoma de Puebla, México (eflores@fcfm.buap.mx)
}

\begin{abstract}
Resumen: El objetivo de este artículo es establecer los elementos del Conocimiento Especializado del Profesor de Matemáticas (MTSK) que se utilizan para darle una atención oportuna a situaciones de dominio afectivo en el aula. Partimos del supuesto de que para atender este tipo de situaciones se necesita conocer la problemática y sus causas, pero también se requiere de distintos conocimientos que le permitan al profesor atender dichas situaciones de manera efectiva. Es así que se realizó una investigación de corte cualitativo a partir de un estudio de caso instrumental. En cuanto a los resultados, se encontró que, en efecto, se emplean algunos elementos del MTSK para atender situaciones afectivas en el aula. Por ejemplo, se emplea el conocimiento sobre estrategias, técnicas, tareas y ejemplos adecuados para favorecer la motivación en los alumnos y la utilidad que estos le atribuyen a las matemáticas.
\end{abstract}

Palavras-Chave: diseño de actividades, dominio afectivo, MTSK

\begin{abstract}
The main of this article is to establish the elements of the Mathematics Teacher's Specialized Knowledge (MTSK) that are used to give timely attention to situations of affective domain in the classroom. We start from the assumption that to deal with this type of situation it is necessary to know the problem and its causes, but it also requires different knowledge that allows the teacher to deal with these situations effectively. Thus, a qualitative investigation was carried out from an instrumental case study. Regarding the results, it was found that indeed some elements of the MTSK are used to address affective situations in the classroom. For example, knowledge about these strategies, techniques, tasks and appropriate examples is used to promote motivation in students and the usefulness they attribute to mathematics.
\end{abstract}

Keywords: activities design, affective domain, MTSK 


\section{Introducción}

A partir de la década de los setenta, un número importante de investigaciones en Didáctica de las Matemáticas comenzaron a centrarse en aspectos afectivos, externos a la disciplina y a la metodología de enseñanza para lograr el aprendizaje de las matemáticas (Gil, Blanco y Guerrero, 2005). Este nuevo enfoque:

Pone de manifiesto que las cuestiones afectivas juegan un papel esencial en la enseñanza y aprendizaje de la matemática, y que algunas de ellas están fuertemente arraigadas en el sujeto y no son fácilmente desplazables por la instrucción. (Gómez-Chacón, 2000, p. 21)

En muchas de estas investigaciones (e.g. Alonso et al., 2005) se han estudiado las repercusiones de factores afectivos en la enseñanza y aprendizaje de las matemáticas pues, como se menciona en Padrón (2005), en el proceso de enseñanza y/o aprendizaje de la Matemática intervienen varios factores que determinan el afecto que se produce hacia esta área del conocimiento o hacia los procesos ligados a ella, los cuales son responsables de muchas de las acciones y comportamientos del sujeto ante la disciplina.

Además, de acuerdo con Gómez-Chacón (2000), el estudiante al aprender matemáticas recibe continuos estímulos asociados a éstas, por ejemplo problemas, actuaciones del profesor, mensajes sociales, etc., ante los cuales reacciona emocionalmente de forma positiva o negativa.

Así, podemos observar que uno de los factores que influye en el afecto que se produce hacia las matemáticas por parte de los alumnos es el profesor $\mathrm{y}$, por lo tanto, todo lo involucrado con su práctica docente. No obstante, él mismo es el responsable de atender las dificultades que se les presentan a sus estudiantes causadas por cuestiones afectivas, las cuales deberían ser atendidas de manera oportuna pues, especialmente en la clase de matemáticas, es muy común encontrar alumnos que muestran reacciones negativas hacia esta área del conocimiento, las cuales evidentemente repercuten negativamente en su aprendizaje.

Por esto, partimos del supuesto de que, para atender este tipo de situaciones relacionadas con cuestiones afectivas, se necesita conocer la problemática y sus causas, pero también se requiere de distintos conocimientos que le permitan al profesor atender dichas situaciones de manera efectiva, favoreciendo el aprendizaje de los alumnos.

A partir de esto, surge el interés de estudiar las relaciones entre los conocimientos que tiene el profesor acerca de los aspectos afectivos que intervienen en la enseñanza y aprendizaje de las matemáticas y los elementos Conocimiento Especializado del Profesor de Matemáticas (MTSK, por sus siglas en inglés) que emplea para atender situaciones de dominio afectivo en el aula. De esta manera, nos hemos planteado las siguientes preguntas de investigación:

1)¿Cómo influye el conocimiento que tienen el profesor acerca de dominio afectivo sobre el conocimiento especializado que emplean para atender situaciones afectivas en el aula?

2)¿Qué elementos del MTSK utiliza el docente al diseñar actividades que atiendan situaciones afectivas en el aula?

\section{Marco teórico}

Debido a que esta investigación está orientada a encontrar los elementos del MTSK que se requieren para atender cuestiones afectivas en el aprendizaje de las matemáticas, nuestro marco teórico se presenta en dos partes. En la primera parte se definen algunos conceptos de dominio afectivo que intervienen en este trabajo y, posteriormente, en la segunda parte se describe el MTSK como un modelo analítico para el estudio del conocimiento del profesor de matemáticas, el cual fue nuestro principal instrumento de análisis en esta investigación.

\section{Dominio Afectivo}

En González et al. (2018) se define el afecto o dominio afectivo como el campo de investigación en matemática educativa dedicado a estudiar algunos aspectos propios del ser humano, como las actitudes, emociones, motivación, creencias, etcétera, los cuales van formando la vida escolar y social de estudiantes y profesores.

Los estudios realizados en este campo han mostrado que el afecto tiene una alta influencia en la motivación académica y en el aprendizaje escolar. Es así como, en años recientes, este enfoque ha tomado rele- 
vancia en tanto que se ha empezado a señalar en los discursos oficiales de organismos internacionales la importancia que tienen los factores afectivos para el aprendizaje, reconociendo que las emociones que desarrolla el alumnado hacia las distintas asignaturas podrían estar relacionadas con el aprendizaje que logra en cada una de ellas (Ursini y Ruiz, 2019).

En particular, en este trabajo se abordan tres factores afectivos en los cuales se centra toda la investigación, los cuales son Percepción de la dificultad de las matemáticas, Desinterés hacia las matemáticas y Valor subjetivo o utilidad de las matemáticas.

Con respecto a la percepción de la dificultad de las matemáticas, Alonso et al. (2005) afirman que las matemáticas tienen características propias como son la abstracción, reflexión, jerarquización, globalización, orden y rigor, las cuales hacen de las matemáticas una disciplina que requiere cierto esfuerzo y el uso de estrategias cognitivas de orden superior para su asimilación; y a ello, se suma el hecho de que los aprendizajes matemáticos son acumulativos, como lo son también las dificultades. Así es como la dificultad que perciben, y más aún, que experimentan los estudiantes en las matemáticas, tiene que ver con la dificultad intrínseca que caracteriza a esta disciplina.

Por otra parte, en lo que respecta al desinterés hacia las matemáticas, González (2005) lo define como la falta de motivación que manifiesta el alumnado en forma de aburrimiento o rechazo por la materia. Este aspecto afectivo tiene gran importancia en el proceso de enseñanza y aprendizaje de las matemáticas pues, como afirma Huertas (1997), la motivación cumple un papel esencial en la enseñanza, ya que es el requisito básico para conseguir el interés por el aprendizaje.

Finalmente, de acuerdo con Sánchez (2019), el valor subjetivo de las matemáticas se refiere a la idea que se tiene acerca de éstas, la cual se forma a partir de las emociones, los sentimientos, las actitudes o los conceptos que el alumno tiene acerca de las matemáticas, de forma que esto incluye la percepción de utilidad de las matemáticas.

\section{Modelo del Conocimiento Especializado del Profe- sor de Matemáticas (MTSK)}

El MTSK es un modelo del conocimiento del profe- sor de matemáticas, el cual parte del supuesto de que para que el profesor lleve a cabo su función (la cual incluye dar clases, planificar las clases y tomarse el tiempo para reflexionar sobre éstas) necesita conocimientos específicos asociados a la enseñanza de las matemáticas (Carrillo-Yañez et al., 2018).

Se trata de una propuesta teórica que modela el conocimiento especializado del profesor de matemáticas $\mathrm{y}$, a su vez, es también una herramienta metodológica que permite obtener información sobre el conocimiento del profesor de matemáticas en su labor profesional y analizarlo a través de sus categorías. El modelo está conformado por dos grandes grupos de conocimiento llamados dominios, los cuales se dividen en tres subdominios cada uno y categorías internas a dichos subdominios. El primer dominio es el Conocimiento Matemático, el cual considera el conocimiento que tiene el profesor de las matemáticas en un contexto escolar. El otro dominio es el Conocimiento Didáctico del Contenido, el cual se refiere al conocimiento que tiene el profesor acerca del contenido matemático como objeto de enseñanza-aprendizaje. Además, en este modelo se considera que las concepciones y creencias del profesor acerca de las matemáticas, su enseñanza y aprendizaje, permean al conocimiento que tiene en cada uno de los subdominios (FloresMedrano et al., 2014).Pasaremos ahora a realizar una breve descripción del contenido de cada uno de los subdominios.

Conocimiento matemático. De acuerdo con Carrillo-Yañez et al. (2018), el conocimiento que tiene el profesor de la disciplina que enseña, es un elemento fundamental que le permite enseñar el contenido de forma coherente y secuenciada, además de que le da la posibilidad de validar sus conjeturas y las de sus alumnos. Así, en el MTSK se consideran tres subdominios que componen al conocimiento matemático: conocimiento de los temas, conocimiento de la estructura matemática y conocimiento de la práctica matemática.

El conocimiento de los temas matemáticos (KoT, por sus siglas en inglés) tiene la finalidad de describir el qué y cómo el profesor de matemáticas conoce los temas que va a enseñar, por lo que en este subdominio se considera el conocimiento que tiene el profesor de 
matemáticas de los contenidos, sus significados y fundamentos. De esta forma, se está considerando tanto el conocimiento del contenido que el alumno debe aprender, como un conocimiento más riguroso que le permite al profesor tener una comprensión más profunda de dichos contenidos. Este subdominio se compone de las categorías: Procedimientos, Definiciones, Propiedades y sus fundamentos, Registros de representación y Fenomenología y aplicaciones

El subdominio del conocimiento de la estructura matemática (KSM, por sus siglas en inglés) considera el conocimiento que le permite al profesor establecer relaciones entre distintos elementos matemáticos, los cuales pueden ser propios del curso que se esté impartiendo, de cursos anteriores, posteriores o de otros niveles educativos. Así, las categorías que lo conforman son: Conexiones basadas en la simplificación, Conexiones basadas en una mayor complejidad, Conexiones auxiliares y Conexiones transversales.

En cuanto al conocimiento de la práctica matemáti$c a$ (KPM, por sus siglas en inglés), en este subdominio se toma en cuenta el conocimiento que tiene el profesor acerca de cómo se generan las matemáticas (por ejemplo qué tipo de pruebas o procedimientos se pueden realizar o cuales son más efectivos para llegar a ciertos resultados). Esto se puede ver con una visión de la Matemática en general o a partir de un caso específico tomando en cuenta las particularidades de un tema, de donde surgen las dos categorías que caracterizan a este subdominio: Prácticas ligadas a la Matemática en General y Prácticas ligadas a una Temática en Matemática.

Conocimiento didáctico del contenido. Este dominio de conocimiento considera el contenido matemático, pero ya no como un conocimiento formal, sino como conocimiento a enseñar-aprender, es decir, que sea accesible y adecuado para los estudiantes de acuerdo al nivel educativo en el que se encuentren. Este conocimiento abona en marcar la diferencia entre una persona que sabe matemáticas y un profesor de matemáticas, pues está estrechamente relacionado con la práctica en el aula. De esta manera, este dominio está constituido por tres subdominios: conocimiento de la enseñanza de las matemáticas, conocimiento de las características de aprendizaje de las matemáticas y conocimiento de los estándares de aprendizaje de las matemáticas.

Respecto al conocimiento de las características de aprendizaje de las matemáticas (KFLM), éste engloba los conocimientos sobre las características propias del aprendizaje de las matemáticas, el cual incluye saber cómo piensan los alumnos y cómo van construyendo sus conocimientos, lo que le permite al profesor comprender los procesos de aprendizaje de los alumnos y, por lo tanto, las fortalezas y dificultades que tiene al aprender matemáticas. De acuerdo con Carrillo-Yañez et al. (2018), las principales fuentes de conocimiento de los docentes, para este subdominio, es su propia experiencia y los resultados de las investigaciones en Educación matemática. Así, las categorías que componen este subdominio son: Teorías del aprendizaje matemático, Fortalezas y debilidades en el aprendizaje de las matemáticas, Maneras en que los alumnos interactúan con el contenido matemático y Aspectos emocionales del aprendizaje de las matemáticas.

El conocimiento de la enseñanza de las matemáticas (KMT) incluye el conocimiento de aspectos inherentes al contenido matemático, pero ahora, como contenido a enseñar, es decir, el conocimiento acerca de las formas o recursos que se pueden emplear para enseñar ciertos contenidos matemáticos. Esto incluye conocer las ventajas y desventajas que los métodos empleados al trabajar matemáticas con los alumnos. Este subdominio comparte fuentes de conocimiento con el KFLM y sus categorías son: Teorías de la enseñanza de las matemáticas, Recursos didácticos (físicos y digitales) y Estrategias, técnicas, tareas y ejemplos.

Finalmente, en el conocimiento de los estándares de aprendizaje de las matemáticas (KMLS) se trata del conocimiento que tiene el profesor acerca de lo que debe aprender el estudiante de acuerdo al nivel educativo al que pertenezca. Este conocimiento orienta el trabajo del docente ya que, por lo general, los niveles de conocimiento que deben alcanzar los alumnos son especificaciones curriculares. Para este subdominio consideramos tres categorías: Resultados de aprendizaje esperados, Nivel esperado de desarrollo conceptual o procedimental y Secuencia de temas. 


\section{Método}

El tipo de investigación que realizamos es este trabajo es de corte cualitativo y mediante estudio de caso. Gómez, Flores y Jiménez (1999), consideran que "el estudio de casos implica un proceso de indagación que se caracteriza por el examen detallado, comprehensivo, sistemático y en profundidad del caso objeto de interés" (p. 92). Además, afirman que un caso puede ser una persona, organización, un programa de enseñanza, un alumno, un profesor, una clase, la práctica de un profesor, etc., y que la única exigencia es que posea algún límite físico o social que le confiera valor o importancia.

En esta investigación usamos un estudio de caso instrumental, ya que éste se emplea cuando se examina un caso particular para proporcionar más información sobre un tema o fenómeno. Así, el caso se convierte en un instrumento para comprender dicho fenómeno y su utilidad radica en la aportación de datos para comprenderlo (Gómez et al., 1999).

En este trabajo, el caso es una profesora de matemáticas que estudió una maestría profesionalizante en Educación Matemática, con la particularidad de que su trabajo de tesis de maestría estuvo enfocado en algunos aspectos de domino afectivo, por lo cual nos da la posibilidad de que tenga cierta sensibilidad hacia ese tipo de temas, sin embargo, no hay garantía de que emplee esos conocimientos en su práctica profesional. De esta manera, nuestro caso se convierte en un instrumento para saber qué elementos del MTSK se emplean al diseñar actividades de aprendizaje que atiendan intencionalmente situaciones relacionadas con cuestiones de dominio afectivo.

Nuestra informante, bajo el seudónimo de Gisela, es maestra de matemáticas con 5 años de experiencia en la docencia, 3 años en secundaria y 2 en nivel medio superior impartiendo clases de estadística y álgebra. Es licenciada en Matemáticas Aplicadas y maestra en Educación Matemática.

Ahora bien, para describir el método y técnicas empleadas en la investigación para la recolección y análisis de datos, se hace una descripción del proceso que seguimos.

En la primera etapa de este estudio comenzamos por seleccionar algunas tesis de maestría realizadas por estudiantes de la Maestría en Educación Matemática (MEM) en la Benemérita Universidad Autónoma de Puebla (BUAP). Dicha selección se hizo principalmente tomando en cuenta la relación que tiene cada trabajo de tesis con algún aspecto afectivo en la enseñanza y aprendizaje de las matemáticas. Además, la posibilidad de establecer contacto con los autores también fue un factor determinante para elegir los trabajos, ya que en una etapa posterior sería necesario establecer un diálogo con ellos.

Una vez seleccionada la tesis de nuestro interés, procedimos a revisarla con la finalidad de identificar los elementos de dominio afectivo involucrados en dicha investigación y la postura de los autores respecto a dichos elementos. Algunas de estas posturas de Gisela encontradas en su tesis, son relevantes para esta investigación y se retoman en el análisis, sin embargo, no se coloca la referencia para mantener la anonimidad de nuestra informante.

El siguiente momento fue la recolección de datos para lo cual procedimos primero a través de una entrevista semiestructurada, ya que ésta nos dio la posibilidad de introducir preguntas adicionales a las consideradas en la guía de entrevista, lo cual permite obtener mayor información sobre los temas deseados. Esto fue con la intención de obtener información acerca de la perspectiva de nuestra informante sobre los elementos de dominio afectivo involucrados en su trabajo de investigación (obtenidos de su tesis) y sobre otros de los que tuviera conocimiento.

Las preguntas de nuestra guía de entrevista se realizaron con base en los elementos de dominio afectivo que se encontraron en el trabajo de tesis de Gisela, a partir de las cuales se estableció un diálogo personal con ella para propiciar una discusión acerca de los elementos de dominio afectivo que conocían. Específicamente, nos interesaba saber cómo concibe dichos elementos, cómo los identifica y cómo sugieren atenderlos en el aula, por lo que se hicieron preguntas de profundización, de ejemplificación y de intervención.

Una vez teniendo la información obtenida en la entrevista, la cual fue recolectada a través de una videograbación, se hizo un análisis temático de dicha información, ya que éste se caracteriza por la necesidad 
que tiene el investigador de establecer las ideas esenciales que guiarán el trabajo de investigación, así como los procedimientos metodológicos y la atención al fenómeno en estudio (Barrera et al., 2012).

Siguiendo la propuesta de Clarke y Braun (2014) para realizar un análisis temático, primero hicimos una identificación e interpretación de patrones de significado en la información obtenida en la entrevista, a partir de lo cual identificamos los temas que guiarían nuestra investigación y revisamos su pertenencia, luego definimos dichos temas y terminamos con la escritura del informe.

Así, con base en el análisis temático realizado, determinamos tres temas de dominio afectivo en los que la profesora mostró mayor énfasis y los cuales guiaron el resto de la investigación. De esta manera los temas de estudio para este trabajo son los siguientes: dificultad en matemáticas, falta de motivación para aprender matemáticas y valor o utilidad de las matemáticas.

Una vez definidos los temas que guiaron esta investigación, el siguiente paso fue solicitar a Gisela un diseño de actividades de aprendizaje que atendiera de manera intencional situaciones afectivas específicas que ella misma rescató y en las que puso mayor interés durante la entrevista.

Una vez teniendo el diseño de las actividades elaborado por la profesora informante, procedimos a analizarlos a través de un análisis Top-Down (de la teoría a los datos), ya que a partir de dichos diseños nos interesaba establecer los elementos del MTSK que se utilizan o se requieren para atender situaciones afectivas en el aula, lo cual se hizo a partir de las categorías del MTSK pues, como sugiere Leal (2020), esta estrategia se caracteriza por recurrir a la teoría sobre el objeto a estudiar y emplearla para analizar la información.

\section{Análisis}

En este apartado presentamos un análisis de la información más relevante para esta investigación, la cual fue obtenida de la entrevista realizada a nuestra informante, Gisela, y de las actividades que diseñó para atender algunos elementos de dominio afectivo. Dicho análisis está enfocado a identificar los componentes del MTSK que la profesora utiliza o requiere para atender determinados aspectos de dominio afectivo en el aula, y se hace en base a las evidencias de conocimiento que nos proporcionó la información recabada.

Cabe destacar, que en este trabajo concebimos el concepto de evidencia de conocimiento como "aquellos elementos que permiten afirmar que un profesor posee, o no, un determinado conocimiento" (Escudero-Ávila et al., 2016. p. 63).

El análisis se aborda en base a los tres temas de dominio afectivo que guiaron este trabajo, los cuales se mencionan en el método. La nomenclatura empleada en los extractos es E para la entrevistadora (primera autora de este trabajo) y $\mathrm{G}$ para Gisela.

\section{Dificultad en matemáticas}

A partir de las respuestas obtenidas en la entrevista que se realizó a Gisela, encontramos que uno de los elementos de dominio afectivo en los que mostró mayor interés, es la dificultad que perciben y experimentan los alumnos al aprender matemáticas, pues fue el elemento de dominio afectivo que más resaltó durante toda la entrevista.

Podemos decir que esto se debe a que ella considera que dicha dificultad es el principal factor que causa actitudes negativas hacia las matemáticas por parte de los estudiantes, pues como afirma literalmente en su trabajo de tesis, "la dificultad de las matemáticas y la vivencia con dicha dificultad son la piedra angular del rechazo hacia dicha asignatura".

Una fuente de dificultad que reconoce Gisela es aquella que proviene del no establecimiento de conceptos previos. Al respecto, ella señala que la dificultad que perciben los alumnos en las matemáticas se debe principalmente a los conceptos o conocimientos previos no aprendidos (a los cuales se refiere como lagunas). Agrega que no solventar dichas lagunas provoca que a los estudiantes se les dificulte más aprender nuevos conceptos matemáticos (véase Extracto 1). Esto va de acuerdo con González (2005), quien dice que uno de los aspectos que influyen en las creencias de los alumnos acerca de la dificultad en matemáticas es la comprensión que alcanza el alumno.

G: Puede que los alumnos tengan mucha dificultad en los conceptos matemáticos en general y que, al llevar lagunas de no entender los conceptos y no 
solventar esas lagunas, entonces se les va a ir complicando más a medida que se les van presentando nuevos conceptos.

\section{Extracto 1}

En particular, Gisela reconoce que la dificultad que los alumnos tienen para operar fracciones aritméticas se convierte en un obstáculo para aprender a operar fracciones algebraicas cuando dicha dificultad no es atendida. Esto se puede observar en el Extracto 2.

G: Por ejemplo, en los alumnos que se les complica bastante la suma de fracciones y si ellos desde la secundaria no solventan este problema, cuando llegamos a álgebra y vemos suma de fracciones algebraicas, obviamente a ellos se les complica demasiado, pero me doy cuenta de que es porque no han comprendido la suma de fracciones aritméticas.

Extracto 2

Esto nos da evidencia de que la profesora tiene conocimiento sobre cuáles son los conocimientos y capacidades previas que debería tener un estudiante para enfrentarse a ciertas tareas. Específicamente, en este caso da evidencia de que reconoce que, para que los alumnos puedan enfrentarse a resolver operaciones entre fracciones algebraicas, es necesario que sepan operar fracciones aritméticas correctamente, lo cual corresponde al conocimiento que tiene la profesora acerca de la secuenciación de estos temas desde cursos anteriores.

Entonces, a partir de lo anterior y con el Extracto 3, podemos decir que Gisela reconoce que el tema de fracciones algebraicas es un tema complicado para los alumnos, y que la vivencia con dicha dificultad los puede conducir a tener actitudes negativas.

G: Fracciones algebraicas es un tema que se ve en Matemáticas 1, en el cual los alumnos llegan a tener dificultades. Entonces yo presiento que este tema les estaría provocando una actitud negativa. [...] Además de que, por otra parte, se les complica mucho pasar del lenguaje común al lenguaje_algebraico. Este es un tema que no entienden mucho los alumnos y yo podría decir que eso podría lle- gar a generales estas conductas negativas [...]. Por ejemplo, ahora que estoy viendo sistemas de ecuaciones por cualquier método, siento que los alumnos sí lo están entendiendo, saben el procedimiento, pero donde se les complica bastante es cuando les pongo un problema verbal y deben formular una ecuación o sistema de ecuaciones para resolverlo. Ese es el único paso donde a ellos se les dificulta demasiado.

Extracto 3

Además, en el Extracto 3 también se puede observar que Gisela reconoce que otra dificultad, que experimentan comúnmente los estudiantes en matemáticas, es el tránsito del lenguaje común al algebraico, e identifica una fortaleza en los estudiantes cuando se trata de hacer procedimientos algorítmicos. Así, los Extractos 2 y 3 nos dan evidencia de que Gisela tiene conocimiento acerca de algunos obstáculos y dificultades comunes de los estudiantes al trabajar con fracciones algebraicas y con problemas verbales, además de que reconoce fortalezas al ver cómo los alumnos dominan los procedimientos algorítmicos. Este conocimiento corresponde al que se considera en la categoría de fortalezas y debilidades asociadas al aprendizaje.

Además, la profesora no solo nos muestra que tiene conocimiento sobre esto, sino que nos comenta cómo lo emplea para atender la dificultad que experimentan sus alumnos. Por ejemplo, ella propone que una forma de atender dicha dificultad es resolver, al inicio de cada clase, ejercicios sencillos que sirvan al alumno para repasar y no olvidar el concepto que se les dificulta, lo cual se observa en el Extracto 4.

E: ¿De qué forma atenderías esta dificultad para evitar que se siga generando más en lo alumnos a la hora de llegar a operaciones con fracciones algebraicas?

G: Lo que yo hago es siempre en los primeros diez minutos, resolver una fracción aritmética, o sea de las sencillas, de distintas maneras, con el mínimo común múltiplo o utilizando las fracciones equivalentes, para que ellos noten que tienes varias formas en las que puedes resolver ese problema y adopten el que mejor entiendan o el que dominen mejor. Pero siempre partir de algo sencillo.

Extracto 4 
Entonces podemos ver que la profesora muestra el conocimiento que tiene acerca de las fortalezas y dificultades asociadas al aprendizaje como un elemento que se puede emplear para atender la dificultad que tienen los alumnos en temas específicos de matemáticas, en este caso, en fracciones algebraicas, para lo cual propone retomar con los alumnos los temas que se les complican las veces que sea necesario, partiendo siempre de ejemplos sencillos.

Además, en el Extracto 4, también podemos observar que la profesora nuevamente evoca al conocimiento que tiene acerca de la secuenciación de los temas. En este caso, de fracciones aritméticas y algebraicas, para atender la dificultad que experimentan los alumnos. Adicionalmente, consideramos que Gisela da evidencia de cómo emplea el conocimiento que tiene acerca de diferentes procedimientos asociados al tema a enseñar para atender la dificultad que presentan los alumnos. Específicamente menciona que, para abordar las operaciones entre fracciones, muestra a sus alumnos varias maneras de resolver los ejercicios (específicamente menciona el método por fracciones equivalentes y utilizando el mínimo común múltiplo) para darles la libertad de utilizar el que más se les facilite. Con esto muestra a los alumnos que no hay una sola forma de solucionar los problemas, por lo que sugiere enseñar a los estudiantes a resolver los ejercicios de formas distintas para que ellos adopten la que más se les facilite y no tengan la limitación de resolverlos de una forma que a ellos se les complique.

\section{Falta de motivación para aprender matemáticas}

Otro elemento de dominio afectivo, del que habló Gisela durante la entrevista, es la falta de motivación para aprender matemáticas por parte de los estudiantes, a lo cual hace referencia en términos de desinterés y apatía, pues como se puede leer en su tesis de maestría ella afirma que la apatía hacia las matemáticas (que se define como la falta de interés) es un factor que influye significativamente en las actitudes que muestra el alumno hacia las matemáticas (tales como rechazo, desgano y aburrimiento) y por lo tanto en su aprendizaje.

A partir de la entrevista con Gisela pudimos percatarnos de que la profesora considera que el tipo de actividades que se eligen para realizar con los estudiantes, influye en las actitudes que ellos muestran hacia las matemáticas, por ejemplo, la falta de motivación para aprender. Pues como se puede observar en el Extracto 5 , ella menciona que la utilización de actividades en las que los alumnos se interesen y que a su vez generen conocimiento, puede contribuir a la disminución de las actitudes negativas hacia las matemáticas por parte de los estudiantes.

E: ¿Qué características crees que deberían tener las actividades didácticas para que las actitudes negativas disminuyan?

G: Realizar quizá un juego o una actividad en lo que ellos se interesen pero que además genere conocimiento.

Extracto 5

Pero más aún, en el diseño de actividades que nos proporcionó Gisela pudimos notar que emplea, de manera inconsciente, algunos elementos del MTSK para atender la falta de motivación de los alumnos en la clase de matemáticas. Por ejemplo, en la Figura 1 podemos observar el problema inicial y la actividad 1

\begin{tabular}{|c|c|}
\hline Sesión 1 & Me podría decir ¿cuál es su nombre? \\
\hline Problema inicial & ¿Cuál es su oficio? \\
\hline Se les pide a los estudiantes que con los medios que dispongan verific & ¿Cuántos años tiene en este oficio? \\
\hline los muros que forman las esquinas del salón de clases forman un ángulo recto. & Cuál es su último grado de estudios? \\
\hline Se espera que los estudiantes propongan varias estrategias, las c & ¿Conoce el teorema de Pitágoras? \\
\hline no, llevar a la solución del problema inicial. & $\begin{array}{l}\text { En caso de conocerlo me podría decir, ¿Qué dice dicho } \\
\text { teorema? }\end{array}$ \\
\hline & ¿En donde utiliza las matemáticas? \\
\hline tro albañil, para ello se sugieren las seguientes preguntas (los equipos pueden & ¿Cuál es el procedimiento para trazar una casa? \\
\hline proponer otras preguntas según lo consideren). & En la construcción, ¿Qué es una escuadra? \\
\hline
\end{tabular}

Figura 1 - Actividad 1 correspondiente a la sesión 1 del diseño didáctico. 
que plantea la profesora para la primera sesión de su diseño de actividades.

Como podemos observar, el problema inicial propuesto por Gisela consiste en pedir a los alumnos que con los medios que dispongan, verifiquen si los muros de las esquinas del salón de clases forman un ángulo recto, con lo que espera que los estudiantes participen activamente y propongan varias estrategias, que puedan o no, llevar a la solución. Luego, propone que los alumnos realicen una entrevista a un trabajador de la construcción para averiguar cómo emplea las matemáticas en su trabajo, especialmente el teorema de Pitágoras.

Luego, en la actividad que se muestra en la Figura 2, la profesora pide a los estudiantes que documenten el proceso que siguen para comprobar si en las esquinas de su casa se forma un ángulo recto (empleando el Teorema de Pitágoras), con la cual ellos pueden aplicar el conocimiento aprendido, pues para este momento la profesora ya les presentó de tema de manera más formal.

Actividad 3
Se les pedirá a los estudiantes que documenten mediante un
video, si las esquinas de los muros de su casa están completa-
mente a escuadra empleando el teorema de Pitágoras.

Figura 2 - Actividad 3 correspondiente a la sesión 4 del diseño didáctico .

Ahora, en la Figura 3 podemos observar que la profesora pide a los estudiantes construir un modelo similar al de la imagen que proporciona, con el cual verifiquen que la suma de los volúmenes de los prismas cuadrangulares formados en los catetos de un triángulo rectángulo, es igual al volumen del prisma que se forma en la hipotenusa (bajo la restricción de que la altura de los tres prismas es la misma). Claramente para la realización de esta actividad interviene el uso de material didáctico.

A partir las actividades presentadas en las Figuras 2, 3 y 4, consideramos que la profesora da evidencia del conocimiento que tiene sobre el tipo de actividades que son adecuadas para abordar el tema del teorema de Pitágoras y a su vez mantener el interés de los alumnos. Esto es porque todas las actividades que se acaban de describir (la medición del ángulo formado por las esquinas del salón de clases, la entrevista realizada al albañil y la construcción del modelo con material didáctico) son actividades donde la participación activa y colaborativa del

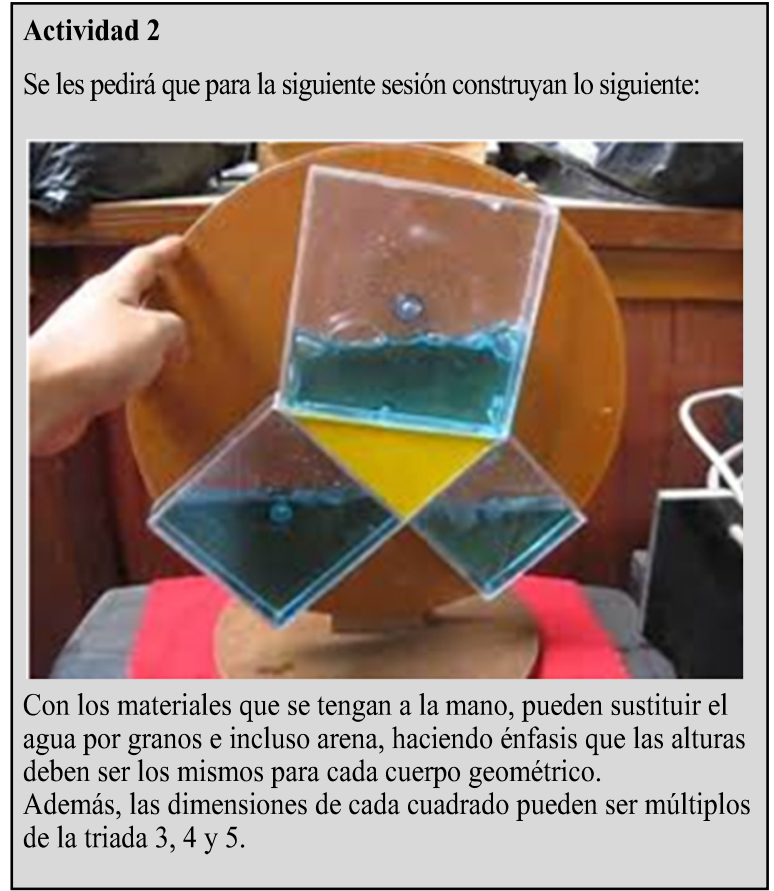

Figura 3 - Actividad 2 correspondiente a la sesión 4 del diseño didáctico.

alumno es indispensable, rompiendo así con el esquema de la enseñanza tradicional. De esta forma, e tipo de actividades y la variedad de estas a lo largo de la secuencia didáctica, mantienen a los alumnos activos en el aprendizaje del tema, por lo que conocimiento que emplea la profesora para atender el desinterés y la apatía de los estudiantes, corresponde a la categoría de estrategias, técnicas, tareas y ejemplos.

Por otro lado, en la actividad 2 de la sesión 2 (véase Figura 4), se muestra que la profesora propone mostrar a los alumnos, a través de un video, la forma en

\begin{tabular}{|l|}
\hline Sesión 2 \\
Actividad 1. \\
Se les pedirá que un integrante del equipo exponga las respues- \\
tas que obtuvieron al resto de sus compañeros. \\
Actividad 2. \\
El profesor les proyectará el siguiente vídeo: \\
https://www.youtube.com/watch?v=K4xmY9NJwbk \\
(Consultado el 8 de noviembre de 2020) \\
Actividad 3. \\
En una plenaria se discutirá si hay respuestas similares tanto de \\
los albiñiles entrevistados como el que aparece en el vídeo. \\
¿Cuáles son esa(s) similitud(es)?
\end{tabular}

Figura 4 - Actividades correspondientes a la sesión 2 del diseño didáctico. 
que los trabajadores de la construcción emplean intuitivamente el teorema de Pitágoras.

Con esta actividad, y con la que se mostró en la Figura 3, donde los alumnos utilizan y manipulan materiales didácticos para la construcción del modelo que se les solicita, la profesora da evidencia del conocimiento que emplea sobre recursos didácticos (físicos $\mathrm{y}$ virtuales), para la enseñanza de este tema y para motivar a sus estudiantes, pues de acuerdo con el estudio realizado por Licea, Frías y Gutiérrez (2017), los alumnos muestran un alto grado de motivación y satisfacción hacia la modalidad apoyada en el uso del video como Recurso Educativo Abierto. Además, como menciona Arrieta (1998), el uso de material en la enseñanza de las matemáticas, favorece la motivación y la actitud positiva hacia la Matemática.

\section{Valor o utilidad de las matemáticas}

De acuerdo a los resultados del trabajo de tesis de Gisela, el valor o utilidad que le atribuye el alumno a las matemáticas es un factor que influye en el desinterés hacia éstas.

Para atender este factor, que evidentemente influye en las actitudes de los estudiantes hacia las matemáticas.

Gisela expresó durante la entrevista que considera importante concientizar a los estudiantes sobre la presencia y la importancia de las matemáticas en nuestro entorno, y que en algunos casos en posible hacerlo a través de problemas de la vida real que puedan solucionarse con los resultados matemáticos que se enseñan. Sin embargo, no da evidencia del conocimiento que tiene acerca de las aplicaciones de algún tema específico. No obstante, en el diseño que nos proporcionó Gisela, la mayor parte de su propuesta gira en torno a la importancia de hacer notar a los estudiantes el uso y aplicaciones del teorema de Pitágoras en la vida real, para lo cual, ella decidió centrarse específicamente en la utilidad de este teorema en el trabajo de construcción.

A partir de esto, podemos decir que la profesora da evidencia de haber empleado el conocimiento que tiene acerca de los usos y aplicaciones del teorema de Pitágoras, el cual corresponde a la categoría fenomenología y aplicaciones. De esta manera, la profesora atiende la falta de utilidad que los alumnos le atribu- yen a las matemáticas en su entono a través de las actividades que le propone a los estudiantes.

Por otro lado, consideramos que con la Actividad 1 de la sesión 4 (véase Figura 5), donde la profesora pide a los estudiantes que verifiquen (empleando el teorema de Pitágoras) si el ángulo formado por una esquina del salón de clases es recto, pone en evidencia el conocimiento que tiene acerca de actividades o tareas adecuadas para que los estudiantes apliquen el teorema de Pitágoras y vean su utilidad en situaciones que se les pueden presentar en su día a día.

\section{Sesión 4}

Para esta sesión se les pedirá una cinta métrica o un flexómetro.

\section{Actividad 1}

Se reunirán en equipos como se formaron en la primera sesión, se les pedirá que elijan alguna de las esquinas del salón o de la barda de la escuela, posteriormente se les pedirá que determinen si es de 90 greados.

Para ello podrá hacer marcas sobre el muro, con tinta que sea fácil de borrar. Además, harán las operaciones matemáticas en su cuaderno.

Finalmente expondrán sus resultados con el resto de sus compañeros, argumentando del por qué no, los muros forman ángulos de 90 grados.

Figura 5 - Actividad 1 correspondiente a la sesión 4 del diseño didáctico.

\section{Conclusiones}

A partir del análisis anterior, podemos concluir que, en general, el conocimiento que tiene la profesora acerca de los aspectos de dominio afectivo que intervienen en el proceso de enseñanza y aprendizaje de las matemáticas, sí influye en el conocimiento que emplea para atender situaciones afectivas en el aula. Pues pudimos percatarnos de que el conocimiento que utiliza depende determinantemente del elemento afectivo que quiera atender y de sus concepciones acerca de cómo influye dicho elemento en el comportamiento de los alumnos hacia las matemáticas, y por lo tanto en su aprendizaje.

En particular, pudimos observar que los elementos del MTSK (en términos de sus categorías) que están relacionados con la atención de la dificultad que los alumnos perciben y experimentan cuando aprenden matemáticas, son el conocimiento que tiene la profesora acerca de la secuenciación de los temas, sobre 
las fortalezas y dificultades asociadas al aprendizaje $\mathrm{y}$ acerca de los diferentes procedimientos que se pueden emplear para abordar el tema de interés. Pues de acuerdo con sus declaraciones, estos conocimientos van trazando la forma y el orden en que se va avanzando en la enseñanza de los temas que a los alumnos se les dificulta.

Por otra parte, con respecto a la falta de motivación que muestran los alumnos para aprender matemáticas, el elemento del MTSK con el que encontramos mayor relación, fue el conocimiento que tiene Gisela acerca del tipo de tareas o actividades que son más adecuadas para abordar un tema en particular, las cuales dependen de la intencionalidad del profesor. Este conocimiento corresponde a la categoría de estrategias, técnicas, tareas y ejemplos y fue utilizado particularmente para elegir el tipo de actividades y tareas que los alumnos deben realizar para aprender el tema en cuestión, las cuales están dotadas de la intencionalidad de mantener el interés de los alumnos para participar activamente y aprender el tema. Además, para atender este elemento de dominio afectivo, también empleó el conocimiento acerca de los recursos didácticos (físicos y virtuales), con lo cual se promueve la motivación de los estudiantes para aprender matemáticas.

Finalmente, también encontramos una relación entre el valor o utilidad que le da al alumno a las matemáticas y algunos elementos del MTSK. En este caso, los conocimientos con los que encontramos mayor relación fueron los conocimientos que tiene la profesora acerca de los diferentes usos y aplicaciones del tema en juego y sobre las actividades y tareas adecuadas para enseñar el tema y a su vez mostrar la utilidad de éste en nuestro entorno. Estos co- nocimientos corresponden a las categorías aplicaciones y fenomenología y estrategias, técnicas, tareas y ejemplos, respectivamente.

Con referencia a los conocimientos que se relacionan con la atención de la dificultad en el aula, se notó gran influencia en la forma en que la profesora lleva la clase, pues de acuerdo a sus declaraciones, en todas sus clases retoma los temas que se les dificultan a los estudiantes para ayudarlos a ir disminuyendo las deficiencias que van arrastrando y que llegan a obstaculizar el avance de su aprendizaje. Para esto, ella emplea el conocimiento que tiene sobre la secuenciación de los temas que aborda y acerca de las fortalezas y debilidades asociadas al aprendizaje de dichos temas. Sin embargo, no se ve la influencia de este conocimiento en el diseño de las actividades para atender dicho elemento de dominio afectivo, por lo que consideramos que es un conocimiento que se requiere para atender la dificultad que experimentan los alumnos en matemáticas y que hace falta gestionar la utilización de este conocimiento en la elaboración de los diseños.

Con respecto a cómo influyen los conocimientos del profesor acerca de dominio afectivo sobre los elementos del MTSK que se emplearon para atender situaciones afectivas en el aula, en este caso se encontró que influyen significativamente en la elección del tipo de actividades que la profesora utiliza en su diseño. Es decir, que el conocimiento que tiene sobre la dimensión afectiva, fue determinante en los conocimientos que empleó acerca del tipo de actividades adecuadas para abordar el tema y a su vez promover la motivación de los estudiantes o contribuir a mejorar el valor o utilidad que le atribuyen los alumnos a éstas. 


\section{Referencias}

Alonso, S. H., Saéz, A. M., \& Picos, A. P. (2005). El perfil emocional matemático como predictor de rechazo escolar: relación con las destrezas y los conocimientos desde una perspectiva evolutiva. Educación Matemática, 17 (2), 89-116.

Arrieta, M. (1998). Medios materiales en la enseñanza de la matemática. Revista de psicodidáctica, (5), 107-114.

Barrera, M. D. M., Tonon, G., \& Salgado, S. V. A. (2012). Investigación cualitativa: el análisis temático para el tratamiento de la información desde el enfoque de la fenomenología social. Universitas humanistica, (74).

Carrillo-Yañez, J., Climent, N., Montes, M., Contreras, LC, Flores-Medrano, E., Escudero-Ávila, D., Vasco, D., Rojas, N., Flores, P., Aguilar González, A., Riveiro, M., \& Muñoz-Catalán, MC (2018). Modelo de conocimientos especializados del profesor de matemáticas (MTSK). Investigación en educación matemática, 20 (3), 236-253.

Clarke, V., \& Braun, V. (2014). Thematic analysis. En A. C. Michalos (Ed.), Encyclopaedia of Quality of Life and Well-Being Research (6626-6628). Springer, Dordrecht, Netherlands: Springer.

Escudero-Ávila, D., Gomes, J., Muñoz-Catalán, M. C., Flores-Medrano, E., Flores, P., Rojas, N., \& Aguilar, A. (2016). Aportaciones metodológicas de investigaciones con MTSK. Reflexionando sobre el conocimiento del professor. Actas de las II Jornadas del Seminario de Investigación de Didáctica de las Matemáticas de la Universidad de Huelva, 60-69.

Flores-Medrano, E., Escudero-Avila, D., Montes, M., Aguilar, A., \& Carrillo, J. (2014). Nuestra modelación del conocimiento especializado del profesor de matemáticas, el MTSK. En J. Carrillo, L.C. Contreras, N. Climent, D. Escudero-Avila, E. Flores-Medrano, \& M. Montes (Eds.). Un marco teórico para el conocimiento especializado del profesor de matemáticas (57-72). Huelva: Universidad de Huelva Publicaciones.

Gil, N., Blanco, L. J., \& Guerrero, E. (2005). El dominio afectivo en el aprendizaje de las Matemáticas. Una revisión de sus descriptores básicos. Revista Iberoamericana de Educación Matemática, 2, 15-32. http:// files.roshimat.webnode.es/200000132-5f344602f0/Documento 10.pdf

Gómez, G. R., Flores, J. G., \& Jiménez, E. G. (1999). Metodología de la investigación cualitativa. Málaga: aljibe.

Gómez-Chacón, I. M. (2000). Matemática emocional. Los afectos en el aprendizaje matemático. Narcea Ediciones.

González, M. S. G., \& Sierra, G. M. (2018). Investigación sobre emociones en la clase de matemáticas. En C. F. Flores, G. M. Sierra, M. S. G. González, J. A. J. López, \& J. C. R. Cruz (Eds.), Investigaciones en dominio afectivo en matemática educativa (19-38).

González, R. M. (2005). Un modelo explicativo del interés hacia las matemáticas de las y los estudiantes de secundaria. Educación Matemática, 17 (1), 107-128.

Huertas, J. A. (1997). Motivación. Querer aprender. AIQUE.

Leal, C. C. F., (2020) Uso del Modelo MTSK para la Caracterización del Conocimiento Especializado del Profesor de Matemáticas en Secundaria: El caso de la Proporcionalidad. Revista Iberoamericana de educación Matemática, (59), 33-63.

Licea, R. A., López Frías, B. S., \& Mortera Gutiérrez, F. J. (2017). El video como Recurso Educativo Abierto y la enseñanza de Matemáticas. Revista electrónica de investigación educativa, 19(3), 92-100.

Padrón, O. J. M. (2005). Dominio afectivo en educación matemática. Paradígma, 26(2), 7-34. https://n9.cl/h5y8d

Sánchez, G. S. (2019). El desinterés hacia las matemáticas en alumnos universitarios de ingeniería y matemáticas: construcción y validación de un instrumento [Tesis de maestría, Benemérita Universidad Autónoma de Puebla].

Ursini, S., \& Ruiz, J. G. S. (Eds.). (2019). Actitudes hacia las matemáticas. Qué son. Cómo se miden. Cómo se evalúan. Cómo se modifican. Universidad Autónoma de México; Facultad de Estudios Superiores. https:// n9.cl/0ns6 